CARTA AL EDITOR

\title{
Psiquiatría de enlace: razones para la consulta, diagnóstico y diferencias entre los pacientes médicos y los quirúrgicos
}

\author{
S. Ruiz-Doblado, ${ }^{*}$ N. Atienza-Martínez ${ }^{* *}$ y J. R. Lacalle-Remigio** \\ *Unidad de Psiquiatria, Hospital Merced, Osuna (Sevilla); **Unidad de Bioestadística, Facultad de Medicina, \\ Universidad de Sevilla, Sevilla, España
}

La psiquiatría de consulta y enlace en el hospital general tiene varios propósitos: ofrecer asistencia de calidad a los pacientes médico-quirúrgicos admitidos en el hospital, enseñar a los profesionales no psiquiatras e investigar sobre aspectos comunes tanto a la psiquiatría como a la medicina [3]. Las razones más frecuentes para las peticiones de consulta fueron la dificultad del médico al hacer un diagnóstico, la enfermedad depresiva y de ansiedad, los intentos suicidas, los síntomas somáticos sin explicación médica, los estados confusionales o agitados, el abuso o la dependencia de sustancias, los problemas en la relación médico-paciente, la negativa a llevar a cabo pruebas complementarias o tratamientos y la existencia de antecedentes psiquiátricos [1]. La discrepancia entre la razón para la consulta y el diagnóstico psiquiátrico es común, normalmente todo se considera depresión y los trastornos mentales orgánicos se diagnostican insuficientemente $[2,4]$. En efecto, a pesar del hecho de que, normalmente, la tasa global de consultas médicas es mayor que las de consultas quirúrgicas, se conoce poco sobre las diferencias en los perfiles de los pacientes que consultan a los médicos y los que consultan a los cirujanos.

Se tomó una muestra simple aleatoria $(\mathrm{n}=160)$ de pacientes médicos (84 casos) y quirúrgicos (76 casos) de la Unidad de Psiquiatría de Enlace del Hospital de la Comarca de Osuna (Sevilla, España). Este hospital público cubre un área rural con una población de 170.000 habitantes aproximadamente. Se registraron las variables sociodemográficas y clínicas para llevar a cabo un estudio descriptivo general, incluidas las razones más frecuentes para la consulta y los diagnósticos de la CIE-10 [5], así como lá identificación o concordancia diagnóstica entre los hallazgos de los médicos y los del psiquiatra. Se evaluaron también las diferencias en el perfil entre los pacientes médicos y los quirúrgicos. Se utilizaron las pruebas 2 y ANOVA, y además un análisis estratificado para ajustar las diferencias en la distribución del género y la edad (prueba de Mantel-Haenszel).

Los resultados más pertinentes del estudio descriptivo y la identificación diagnóstica por los médicos no psiquiatras se pueden encontrar en la Tabla I. Las razones más frecuentes para la consulta y los diagnósticos psiquiátricos fueron similares a los encontrados en otros estudios [2]. Los trastornos mentales orgánicos (F00-F09, sobre todo el delirium) predominaron en nuestra serie. Es corriente la identificación insuficiente del grupo de los trastornos mentales orgánicos (F00-F09) [2], lo que indica la dificultad que los médicos no psiquiatras tienen al reconocer las causas orgánicas subyacentes a los estados confusionales

Ruiz-Doblado S, Atienza-Martínez N, Lacalle-Remigio JR. Liaison-psychiatry: reasons for consultation, diagnosis, and differences between medical and surgical patients. Eur Psychiatry 1999; 14: 107-8. 
Tabla I. Resultados.

Razones más frecuentes para la consulta (personal no psiquiátrico)

$\begin{array}{lr}\text { Depresión } & 19,4 \%\end{array}$

Trastorno del comportamiento debido a confusión o agitación $\quad \therefore 16,3 \%$

$\begin{array}{ll}\text { Control del tratamiento/historia psiquiátrica } & 14,4 \%\end{array}$

Problemas para alcanzar un diagnóstico diferencial $\quad \ldots . \quad \cdots$

Problemas en la relación entre el médico y el paciente, con la información, negativa a llevar a cabo
pruebas o tratamientos

$\begin{array}{ll}\text { Abuso o dependencia de sustancias } & 8,8 \%\end{array}$

Grupo diagnóstico de la CIE-10 de mayor prevalencia (diagnóstico psiquiâtrico) [5]

Trastornos mentales orgánicos (F00-F09) $\quad 28,8 \%$

$\begin{array}{lr}\text { Trastornos de adaptación (F43.2) } & 22,0 \%\end{array}$

Trastornos del humor (afectivos) (F30-F39) $\quad 13,2 \%$

$\begin{array}{ll}\text { Abuso/dependencia de sustancias (F10-F19) } & 12,5 \%\end{array}$

Concordancia entre el personal no psiquiátrico y el psiquiátrico

Abuso/dependencia de sustancias (F10-F19)

sensibilidad $73,7 \%$

Trastornos del humor (afectivos) (F30-F39)

sensibilidad $63,2 \%$

Trastornos neuróticos y relacionados (F40-F48)

sensibilidad $55,3 \%$

Trastornos mentales orgánicos (F00-F09) sensibilidad $45,7 \%$

agudos. Los pacientes del grupo de trastornos neuróticos-secundarios a situaciones estresantes-somatomorfos de la CIE-10 eran los más problemáticos para los médicos al intentar alcanzar un diagnóstico diferencial (20,7\% del número total de neuróticos). Hubo también problemas con la relación entre el médico y el paciente en este grupo, así como negativas a llevar a cabo pruebas diagnósticas o tratamientos $(13,8 \%$ del número total de neuróticos).

Con respecto a las diferencias entre los pacientes médicos y quirúrgicos; hubo más pacientes médicos con trastornos relacionados con sustancias (F10-F19) y trastornos del humor (F30-F39), mientras que en el grupo quirúrgico predominaron los trastornos neuróticos, secundarios a situaciones estresantes y somatomorfos (F40-48). La diferencia no era estadísticamente significativa $(P=0,06)$, excepto cuando se estratificaba para los pacientes jóvenes. Esto se debía sobre todo a los trastornos relacionados con sustancias, en lo que se refería a los pacientes médicos, y a los trastornos de ansiedad y los trastornos mentales orgánicos con los pacientes quirúrgicos. Se ha señalado también que la cirugía se relaciona con clases de ansiedad diferentes (antes, durante y después de la operación). La preocupación por no valerse por sí mismo y tener que depender de otros, el temor a la muerte, el dolor, la anestesia, la pérdida de partes o funciones corporales, la recuperación postoperatoria, etc, representan razones comunes para los estados de ansiedad. Con frecuencia, son comunes además los estados confusionales post-operatorios. Este hecho podría explicar también las diferencias de género entre los pacientes médicos y los quirúrgicos: el abuso de sustancias es más común en los hombres y los trastornos neuróticos-de ansiedad en las mujeres.

\section{BIBLIOGRAFÍA}

1 Cirera-Costa E. Introducción. In Rojo-Rodes JE, CireraCosta $\mathrm{E}$ eds. Interconsulta psiquiátrica. Barcelona: Masson; 1997.p. 3-20.

2 Clarke DM, Smith GC. Consultation-liaison psychiatry in general medical units. Aust N Z J Psychiatry 1995; 29: 424-32.

3 Lipowski ZJ. Consultation-liaison psychiatry: the first half century. Gen Hosp Psychiatry 1986; 8: 305-15.

4 Margolis RL. Non-psychiatrist house staff frequently misdiagnose psychiatric disorders in general hospital inpatients. Psychosomatics 1994; 35: 485-91.

5 World Health Organization (WHO). Trastornos Mentales y del Comportamiento: criterios diagnósticos de investigación, 10ª edición (ICD-10). Madrid: Meditor; 1992. 